\title{
Another string to the bow
}

Many of the regulators of the homologydirected DNA-repair pathway, homologous recombination (HR), such as BRCA2 and the RecQ helicases, WRN and BLM, are often associated with cancer owing to unproductive HR repair and genomic instability. Reporting in Cell, Simon Boulton and colleagues have identified a new HR gene in humans that may also be deregulated in cancer.

In Saccharomyces cerevisiae the helicase Srs2 antagonizes the initial steps of HR, and srs 2 mutants exhibit spontaneous recombination and sensitivity to DNA-damaging agents that produce $\mathrm{HR}$ substrates, similarly to the loss of other HR genes. The homologue of Srs2 in higher eukaryotes has not yet been identified, so Barber and colleagues conducted a candidatebased synthetic lethality screen in Caenorhabditis elegans. They identified a mutant of $\mathrm{F} 25 \mathrm{H} 2.13$ - which they named rtel-1 - as a putative Srs2 homologue. The single mutants were viable but their life cycle was retarded and they exhibited pheno-

types that were consistent with genomic instability arising from unrepaired DNA damage. Moreover, double mutants with ablated

HR-associated DNAdamage repair or helicase genes showed significant accummulation of foci that contained the HR protein RAD-51. Indeed, consistent with Srs2, the authors found that rtel-1 mutants exhibited an approximately fourfold increase in meiotic crossing over (for which HR is required) and sensitivity to DNA-damaging agents that cause intrastrand crosslinks (which are substrates for HR), indicating that RTEL-1 antagonizes HR.
So, is Srs 2 conserved in humans? Barber and colleagues identified RTEL1 in humans and used small interfering RNA in HeLa cells to show that HR increased by approximately fourfold and cells were sensitive to intrastrand crosslink-inducing drugs, indicating that RTEL1 suppresses HR in humans. Moreover, using an in vitro $\mathrm{HR}$ assay, they showed that RTEL1 reversed an early stage in HR. Therefore, although there are some differences, RTEL1 in both humans and C. elegans seems to be a functional analogue of Srs2.

RTEL1 is within a locus containing three other genes that is overexpressed in some gastrointestinal tumours, and the authors posit that increased expression of RTEL1 could suppress productive $H R$, thereby mimicking the effects of oncogenic HR mutants. Indeed, analysis of tumour samples might reveal a common trait of RTEL1 deregulation in cancer.

Gemma K. Alderton

ORIGINAL RESEARCH PAPER Barber, L. J. et al. RTEL1 maintains genomic stability by suppressing homologous recombination. Cell 135, 261-271 (2008)

FURTHER READING Hickson, I. D. RecQ

helicases: caretakers of the genome. Nature Rev.

Cancer 3, 169-178 (2003) 